\title{
EXISTENCE AND REGULARITY FOR A CURVATURE DEPENDENT VARIATIONAL PROBLEM
}

\author{
JOCHEN DENZLER
}

ABSTRACT. It is proved that smooth closed curves of given length minimizing the principal eigenvalue of the Schrödinger operator $-\frac{d^{2}}{d s^{2}}+\kappa^{2}$ exist. Here $s$ denotes the arclength and $\kappa$ the curvature. These minimizers are automatically planar, analytic, convex curves. The straight segment, traversed back and forth, is the only possible exception that becomes admissible in a more generalized setting. In proving this, we overcome the difficulty from a lack of coercivity and compactness by a combination of methods: geometric improvement algorithm, relaxed variational problem, asymptotic analysis, and strong variations.

\section{IntRoduction AND OUTLINE}

Given a closed curve $\gamma: s \mapsto \boldsymbol{x}(s)$ of length $2 \pi$ in $\mathbb{R}^{n}$, we consider the principal eigenvalue $\lambda$ of the Schrödinger operator $\mathbf{H}=-\frac{d^{2}}{d s^{2}}+\kappa^{2}$ on the curve; here $s$ is the arclength and $\kappa(s)$ is the curvature $\left|\boldsymbol{x}^{\prime \prime}(s)\right|$. Considering $\lambda$ as a function of the curve, we ask what (if any) is the minimal possible value of $\lambda$, and for which curves it is taken on. Fixing the length to $2 \pi$ is no loss of generality, since the problem is homogeneous with respect to dilations.

A natural conjecture is that the minimal $\lambda$ is 1 ; this value is indeed the principal eigenvalue of $\mathbf{H}$ for the unit circle. But it is known to also be the principal eigenvalue of $\mathbf{H}$ for a certain 1-parameter family of ovals (convex planar curves); this family connects the unit circle to a limiting case we call 'di-gon': a straight segment of length $\pi$ traversed back and forth.

The Oval Conjecture states that this family of ovals does consist of minimizers. A strengthened version would stipulate that these are the only minimizers. So far, the Oval Conjecture is open. It has been shown by Burchard and Thomas [3] that this family consists of relative minimizers (in the sense of weak minimizers: minimal among competitors in a neighborhood defined by a strong topology). It is also easy to see (from the equivalent version (2.6) below and the fact that each component $\psi_{i}$ there must have a zero) that $\lambda \geq \frac{1}{4}$ in any case, and that $\lambda \geq 1$ for curves enjoying a point symmetry. An elegant elementary argument (for convex planar curves) by Linde [4] raised the previously known lower bound $\lambda \geq \frac{1}{2}$ [1] to

Received by the editors December 7, 2012.

2010 Mathematics Subject Classification. Primary 53A04; Secondary 49J45, 49N60, 49R50.

The author gratefully acknowledges repeated useful discussions with Almut Burchard. She inspired the research and was available to discuss and critique progress. Some of her ideas enter in the arguments, as outlined in the main text. This reseach was partly supported by a grant from the Simons Foundation (\#208550). The hospitality of the CRM Université de Montréal during the workshop on Geometry of Eigenfunctions June 4-8, 2012 was a boost to this research, as was a Faculty Development Leave ('Sabbatical') granted by the University of Tennessee during Spring 2012, and the hospitality of Karlsruhe Institute of Technology during said leave. 
some quantity $\lambda_{*} \approx 0.6085$, and also established $\lambda \geq 1$ for a certain class of ovals defined in geometric terms.

An interesting aspect of this variational problem is that a positive answer to the above conjecture (in 2 dimensions) implies that the best constant $L$ in the 1 dimensional Lieb-Thirring inequality $\lambda \leq L \int_{\mathbb{R}} V_{-}^{3 / 2}$ for the Schrödinger operator $-\frac{d^{2}}{d x^{2}}+V(x)$ with a single bound state also applies to potentials with two bound states. See [1] for details. (Lest a wrong impression be created by omission, it may be mentioned here that the connection in [1] does not identify $V$ with $\kappa^{2}$.)

Moreover, Bernstein and Breiner 2 have established a connection between the Oval Problem and a minimization property of the catenoid. Namely, they show that among all minimal surfaces of the topological type of an annulus that connect two parallel planes in $\mathbb{R}^{3}$, the marginally stable catenoid has the smallest area. One of the proofs they provide relies on the assumption that the oval conjecture holds; however they also give a proof that is independent of the oval conjecture.

The degenerating family of ovals with common principal eigenvalue 1 shows a lack of compactness (even of coercivity) in the problem. Sublevel sets $\{\gamma \mid \lambda(\gamma) \leq a\}$ for $a \geq 1$ lack any a-priori bounds on curvature, even in an $L^{p}$ norm with $p>1$. Therefore they fail to be compact in any plausible topology for the problem. This is an obstruction to an existence proof by direct methods, and is also an obstruction to a regularity theory for minimizers, should they indeed exist.

Despite these difficulties, we prove in this paper existence and regularity for minimizers. Incidentially, this shows that Linde's assumptions in his proof that $\lambda \geq \lambda_{*} \approx 0.6085$ for planar convex curves are no loss of generality in the full problem.

Specifically we prove:

Theorem 1.1. Among all closed $W^{2,2}$ (or $C^{2}$ ) curves of length $2 \pi$ in $\mathbb{R}^{n}$, there exists one that minimizes the principal eigenvalue of the operator $\mathbf{H}=-\frac{d^{2}}{d s^{2}}+\kappa^{2}$, where $s$ is the arclength and $\kappa$ is the curvature. Minimizing curves are planar convex analytic curves with strictly positive curvature.

To achieve this goal, we first define a relaxed variation problem for which some compactness is restored, allowing for an existence proof. We then show, comparatively easily, that the singular set of a potential minimizer can consist of at most two points. An explicit descent algorithm shows that any minimizer must be planar and convex. The Euler-Lagrange equations of the variation problem play only a weak role in this argument: we basically exploit them for standard regularity results for solutions to ODEs. Subsequently, we will also use them for local asymptotic results near singularities. Beyond this use, the Euler-Lagrange equations so far seem to be of very limited use in this problem. Indeed, they appear to allow for chaotic dynamics (loosely speaking, and judging merely on the basis of some numerical experiments). In particular, planarity is not a feature that would follow from the Euler-Lagrange dynamics. However, Euler-Lagrange dynamics does imply that extremal curves lie in a space of dimension at most 3 . While this fact is not essential for our argument, it does simplify the reasoning a bit.

The arguments outlined so far still amount only to a partial regularity result, leaving the possibility of 'D-shaped' minimizers. We call a minimizing curve Dshaped if it has one or two singular points, where the curvature fails to be $C^{2}$ (possibly even allowing for a corner there), and where a straight segment connects 
the two singular points; the case of only one singular point is included, with the straight segment then omitted.

We will rule out D-shaped minimizers by a combination of two tools, namely: asymptotic analysis near the singularity, following from the Euler-Lagrange equation (albeit with some a-priori input derived from minimality), and some further curve surgery argument that applies to minimizers only. These latter surgery arguments are of a local nature (i.e., variations supported on small intervals) and amount to strong variations not seen by the Euler-Lagrange equations. In this context, strong variations are those that are small in the $C^{1}$ norm, but large in the $C^{2}$ norm of the curve.

In the relaxed variation problem, the di-gon is a possible minimizer (that is ruled out by the $W^{2,2}$ assumption on the curve). Should the minimum of the principal eigenvalue be 1 as conjectured, then the above-mentioned explicitly known ovals that have the di-gon as a limiting case are regular minimizers (we do not know if they are the only ones). Should the minimum be less than 1 , the sole possible exception to regularity for the relaxed variation problem doesn't apply at all.

For the detailed proof, the above theorem is split up into Theorems 3.1, 3.3, and 3.4 below, which are proved separately.

While the oval conjecture remains open, certainly the existence theorem and geometric properties of minimizers proved here should be expected to limit the quest for minimizers in a useful manner.

\section{DeFinition of ORIGINAL AND RELAXED VARIATION PROBLEMS}

We write $S^{1}=\mathbb{R} / 2 \pi \mathbb{Z}$ for the unit circle and assume we have a closed rectifiable curve $\gamma$ parametrized by arclength $s$, and with reasonably defined curvature. So $\gamma$ is given by $\boldsymbol{x} \in C^{2}\left(S^{1} \rightarrow \mathbb{R}^{n}\right)$, or possibly $\boldsymbol{x} \in W^{2,2}\left(S^{1} \rightarrow \mathbb{R}^{n}\right)$, subject to $\left|\boldsymbol{x}^{\prime \prime}\right| \equiv 1$. We let $\kappa(s):=\left|\boldsymbol{x}^{\prime \prime}(s)\right|$ denote the curvature. The principal eigenvalue $\lambda$ of the operator $\mathbf{H}=-\frac{d^{2}}{d s^{2}}+\kappa^{2}(s)$ is defined by the Rayleigh-Ritz variation problem

$$
\begin{aligned}
& \lambda(\gamma):=\min \left\{\int_{0}^{2 \pi}\left(\phi^{\prime 2}(s)+\kappa^{2}(s) \phi^{2}(s)\right) d s \mid\right. \\
&\left.\phi \in W^{1,2}\left(S^{1}\right), \int_{0}^{2 \pi} \phi^{2}(s) d s=1\right\},
\end{aligned}
$$

which is known to have a minimizer that is unique up to sign; we can choose $\phi \geq 0$ with no loss of generality. Then, with $\kappa^{2} \in L^{1}$, the eigenfunction $\phi$ lies in $W^{2,1} \subset C^{1}$ and satisfies $-\phi^{\prime \prime}+\kappa^{2} \phi=\lambda \phi$. Nonnegative solutions of this equation cannot vanish at all unless they vanish identically; this follows from a version of the uniqueness theorem for initial value problems for linear ODEs with $L^{1}$ coefficients. We are studying the minimization problem

$$
\begin{aligned}
\inf \{\lambda(\gamma) \mid \gamma & : s \mapsto \boldsymbol{x}(s) \\
& \text { with } \left.\boldsymbol{x} \in W^{2,2}\left(S^{1} \rightarrow \mathbb{R}^{n}\right),\left|\boldsymbol{x}^{\prime}\right| \equiv 1,\left|\boldsymbol{x}^{\prime \prime}(s)\right|=\kappa(s)\right\}
\end{aligned}
$$

These two can be combined into the variation problem

$$
\begin{aligned}
& I[\boldsymbol{x}, \phi]:=\int_{0}^{2 \pi}\left(\phi^{\prime 2}+\left|\boldsymbol{x}^{\prime \prime}\right|^{2} \phi^{2}\right) d s, \\
& \inf \left\{I[\boldsymbol{x}, \phi]\left|\boldsymbol{x} \in W^{2,2}\left(S^{1} \rightarrow \mathbb{R}^{n}\right), \phi \in W^{1,2}\left(S^{1}\right),\right| \boldsymbol{x}^{\prime} \mid \equiv 1,\|\phi\|_{L^{2}}^{2}=1\right\} .
\end{aligned}
$$


As outlined, it is no loss of generality also to require $\phi>0$ in (2.3). Introducing the function $\boldsymbol{\psi}:=\boldsymbol{x}^{\prime} \phi \in W^{1,2}\left(S^{1} \rightarrow \mathbb{R}^{n}\right)$, there is an equivalent formulation that was already used in [3]. The condition that $\boldsymbol{x}$ represents a closed curve requires $\boldsymbol{x}^{\prime}=\boldsymbol{\psi} /|\boldsymbol{\psi}|$ to integrate to 0 .

Definition 2.1. The classical oval problem in curve coordinates is the variation problem (2.3). The classical oval problem in harmonic coordinates is

$$
\begin{aligned}
& I[\boldsymbol{\psi}]:=\int_{0}^{2 \pi}\left|\boldsymbol{\psi}^{\prime}\right|^{2}(s) d s, \\
& \inf \left\{\left.I[\boldsymbol{\psi}]\left|\boldsymbol{\psi} \in W^{1,2}\left(S^{1} \rightarrow \mathbb{R}^{n} \backslash\{\boldsymbol{0}\}\right), \int_{0}^{2 \pi}\right| \boldsymbol{\psi}\right|^{2} d s=1, \int_{0}^{2 \pi} \frac{\boldsymbol{\psi}}{|\boldsymbol{\psi}|} d s=\mathbf{0}\right\} .
\end{aligned}
$$

The following establishes the equivalence of (2.3) and (2.4) and is routine to check:

Lemma 2.2. If $(\boldsymbol{x}, \phi)$ is in the domain of (2.3) and $\phi>0$, then $\boldsymbol{\psi}:=\boldsymbol{x}^{\prime} \phi$ is in the domain of (2.4). Conversely, if $\boldsymbol{\psi}$ is in the domain of (2.4), then $\phi:=|\boldsymbol{\psi}|$ and $\boldsymbol{x}:=\int \boldsymbol{\psi} /|\boldsymbol{\psi}|$ (with any choice of the constant of integration) provide an $(\boldsymbol{x}, \phi)$ in the domain of (2.3). It holds that $I[\boldsymbol{x}, \phi]=I[\boldsymbol{\psi}]$.

Now in (2.3), the functional does not control $\int\left|\boldsymbol{x}^{\prime \prime}\right|^{2}$. In (2.4), the condition that $\psi$ doesn't vanish anywhere is not stable under any sensible convergence notion for $\psi$, in particular not weak or strong $W^{1,2}$ convergence. It does make sense to enlarge the domain of (2.4) by requiring $\psi$ to vanish only on a set of measure zero, but this does not mend the loss of stability under convergence. Doing so allows curves $\boldsymbol{x}$ with non- $L^{2}$ curvature, or even with corners, provided $\phi$ vanishes in those points.

Given $\boldsymbol{x} \in W^{1, \infty}\left(S^{1}\right)$, we define with the obvious understanding of an interval $[s-\varepsilon, s+\varepsilon]$ as a subset of $S^{1}$, the exceptional set

$$
E[\boldsymbol{x}]:=\left\{s \mid \boldsymbol{x} \notin W^{2,2}[s-\varepsilon, s+\varepsilon] \text { for any } \varepsilon\right\} .
$$

Given $\boldsymbol{\psi} \in W^{1,2}\left(S^{1} \rightarrow \mathbb{R}^{n}\right)$, we define the zero set

$$
Z[\boldsymbol{\psi}]:=\{s \mid \boldsymbol{\psi}(s)=\mathbf{0}\} .
$$

By definition of $E$ and continuity of $\boldsymbol{\psi}$ respectively, $E[\boldsymbol{x}]$ and $Z[\boldsymbol{\psi}]$ are closed sets; $\kappa$ is defined exactly on the complement of $E[\boldsymbol{x}]$.

Definition 2.3. The relaxed oval problem in curve coordinates is

$$
\left\{\begin{array}{c}
I[\boldsymbol{x}, \phi]:=\int_{S^{1} \backslash E[\boldsymbol{x}]}\left(\phi^{2}+\left|\boldsymbol{x}^{\prime \prime}\right|^{2} \phi^{2}\right) d s \\
\text { for } \boldsymbol{x} \in W^{1, \infty}\left(S^{1} \rightarrow \mathbb{R}^{n}\right), \phi \in W^{1,2}\left(S^{1}\right), \\
\inf \left\{I[\boldsymbol{x}, \phi]|| \boldsymbol{x}^{\prime} \mid \equiv 1,\|\phi\|_{L^{2}}^{2}=1, \phi=0 \text { on } E[\boldsymbol{x}]\right\} .
\end{array}\right.
$$

We let $\sigma$ denote the 1-dimensional Lebesgue measure and define $\operatorname{sgn} \boldsymbol{\psi}:=\boldsymbol{\psi} /|\boldsymbol{\psi}|$ provided $\boldsymbol{\psi} \neq \mathbf{0}$, and $\operatorname{sgn} \mathbf{0}:=\mathbf{0}$. Then the relaxed oval problem in harmonic coordinates is

$$
\begin{aligned}
& \inf \left\{I[\boldsymbol{\psi}]:=\int_{0}^{2 \pi}\left|\boldsymbol{\psi}^{\prime}\right|^{2}(s) d s \mid\right. \\
& \left.\quad \boldsymbol{\psi} \in W^{1,2}\left(S^{1} \rightarrow \mathbb{R}^{n}\right), \int_{0}^{2 \pi}|\boldsymbol{\psi}|^{2} d s=1,\left|\int_{0}^{2 \pi} \operatorname{sgn} \boldsymbol{\psi} d s\right| \leq \sigma(Z[\boldsymbol{\psi}])\right\} .
\end{aligned}
$$

We refer to this last constraint as the weak loop condition. 
Note that the given domain in (2.5) ascertains that the integral $I[\boldsymbol{x}, \phi]$ in the functional is defined in the extended sense, but does not guarantee a finite value for it.

We now claim

Lemma 2.4. If $(\boldsymbol{x}, \phi)$ is in the domain of (2.5) with $I[\boldsymbol{x}, \phi]<\infty$, then $\boldsymbol{\psi}:=\boldsymbol{x}^{\prime} \phi$ is in the domain of (2.6), $Z[\boldsymbol{\psi}] \supset E[\boldsymbol{x}]$, the functional is the same, and $I[\boldsymbol{\psi}]=I[\boldsymbol{x}, \phi]$.

Conversely, assume that $\boldsymbol{\psi}$ is in the domain of (2.6), let $\phi:=|\boldsymbol{\psi}|$ and $\boldsymbol{y}:=$ $\int \operatorname{sgn} \boldsymbol{\psi}$ (with any choice of the constant of integration). Then $\boldsymbol{y}$ describes a (not necessarily closed) rectifiable curve segment of length $\sigma\left(Z[\boldsymbol{\psi}]^{c}\right)$ that can be extended to a closed curve $\boldsymbol{x}$ of length $2 \pi$, such that $E[\boldsymbol{x}] \subset Z[\boldsymbol{\psi}],(\boldsymbol{x}, \phi)$ is in the domain of (2.3), and $I[\boldsymbol{\psi}]=I[\boldsymbol{x}, \phi]$.

Proof. If $(\boldsymbol{x}, \phi)$ is in the domain of (2.5), then $E[\boldsymbol{x}]^{c}$ cannot be empty because this would entail incompatible constraints on $\phi$. By definition of $E[\boldsymbol{x}]$, we have $\boldsymbol{x} \in W_{\mathrm{loc}}^{2,2}\left(E[\boldsymbol{x}]^{c}\right)$, hence $\boldsymbol{\psi}=\boldsymbol{x}^{\prime} \phi \in W_{\mathrm{loc}}^{1,2}\left(E[\boldsymbol{x}]^{c}\right)$. Since $\left|\boldsymbol{\psi}^{\prime}\right|^{2}=\left|\phi^{\prime}\right|^{2}+\left|\boldsymbol{x}^{\prime \prime}\right|^{2} \phi^{2}$, the finiteness of the functional in (2.5) implies $\boldsymbol{\psi} \in W^{1,2}\left(E[\boldsymbol{x}]^{c}\right)$. So $\boldsymbol{\psi}$ is continuous on $E[\boldsymbol{x}]^{c}$. Moreover $|\boldsymbol{\psi}|=|\phi|$ is continuous on $S^{1}$ and vanishes on $E[\boldsymbol{x}]$. So $\boldsymbol{\psi} \in C^{0}\left(S^{1}\right)$ and $\boldsymbol{\psi}=\mathbf{0}$ on $E[\boldsymbol{x}]$. The extension of $\boldsymbol{\psi} \in W^{1,2}\left(E[\boldsymbol{x}]^{c}\right)$ by $\mathbf{0}$ to $S^{1}$ is therefore in $W^{1,2}\left(S^{1}\right)$. We have also obtained $Z[\boldsymbol{\psi}] \supset E[\boldsymbol{x}]$ in the process and only have to verify the weak loop condition. But $\left|\int_{S^{1}} \operatorname{sgn} \boldsymbol{\psi}\right|=\left|\int_{Z[\boldsymbol{\psi}]^{c}} \boldsymbol{x}^{\prime}\right|=\left|-\int_{Z[\boldsymbol{\psi}]} \boldsymbol{x}^{\prime}\right| \leq \sigma(Z[\boldsymbol{\psi}])$.

For the converse statement, assume $\boldsymbol{\psi}$ is in the domain of (2.6). Clearly $\psi:=|\boldsymbol{\psi}|$ has $L^{2}$-norm 1 , is continuous on $S^{1}$ and vanishes on $Z[\boldsymbol{\psi}]$, because $\boldsymbol{\psi} \in W^{1,2}\left(S^{1}\right)$ has these properties. Also $\psi \in W^{1,2}\left(Z[\boldsymbol{\psi}]^{c}\right)$. Hence $\psi \in W^{1,2}\left(S^{1}\right)$ with $\psi, \psi^{\prime}=0$ on $Z[\boldsymbol{\psi}]$. The function $\phi$ will shortly arise from $\psi$ by extension and reparametrization.

Next, $\boldsymbol{y}(t):=\int_{0}^{t} \operatorname{sgn} \boldsymbol{\psi}(t) d t$ defines a function in $W^{1, \infty}\left([0,2 \pi] \rightarrow \mathbb{R}^{n}\right)$ with $\left|\frac{d}{d t} \boldsymbol{y}\right|=1$ on $Z[\boldsymbol{\psi}]^{c}$ and $\left|\frac{d}{d t} \boldsymbol{y}\right|=0$ on $Z[\boldsymbol{\psi}]$. This function $\boldsymbol{y}$ represents a curve segment of length exactly $\sigma\left(Z[\psi]^{c}\right)$, with the arc length parameter not $t$, but $s:=$ $\int_{0}^{t}|\operatorname{sgn} \boldsymbol{\psi}(t)| d t$. We estimate $|\boldsymbol{y}(2 \pi)-\boldsymbol{y}(0)|=\left|\int_{0}^{2 \pi} \boldsymbol{y}^{\prime}(t) d t\right|=\left|\int_{0}^{2 \pi} \operatorname{sgn} \boldsymbol{\psi}(t) d t\right| \leq$ $\sigma(Z[\boldsymbol{\psi}])$, where the weak loop condition from (2.6) was used in the last step. So the curve segment $\boldsymbol{y}$ can be extended to a closed curve $\gamma$ by adding a smooth curve of length exactly $\sigma(Z[\boldsymbol{\psi}])=: \ell$ with $t \in[2 \pi, 2 \pi+\ell]$ the arclength parameter on this segment.

We extend $\psi$ by 0 on this extra piece of curve. So $\boldsymbol{y}$ and $\psi$ are now functions of $t \in[0,2 \pi+\ell]$. Reparametrizing them to arclength $s \in[0,2 \pi]$ according to $s=\int_{0}^{t}|\operatorname{sgn} \psi(t)| d t$ for $t \in[0,2 \pi]$, and $s=t-\ell$ for $t \in[2 \pi, 2 \pi+\ell]$, we get $\boldsymbol{x}(s):=y(\mathbf{t}), \phi(s):=\psi(t)$.

We have $\int\left(\frac{d \phi}{d s}\right)^{2} d s=\int\left(\frac{d \psi}{d t}\right)^{2} d t$ and $\int \phi(s)^{2} d s=\int \psi(t)^{2} d t$, since $\frac{d s}{d t}=1$ wherever $\phi \neq 0$. Since $\boldsymbol{x} \in W_{\mathrm{loc}}^{2,2}\left(Z[\boldsymbol{\psi}]^{c}\right)$, we know $E[\boldsymbol{x}] \subset Z[\boldsymbol{\psi}]$, as far as $s \in\left[0, \sigma\left(Z[\boldsymbol{\psi}]^{c}\right)\right]$ is concerned. Clearly $\left.s \in\right] \sigma\left(Z[\boldsymbol{\psi}]^{c}\right), 2 \pi[$ does not contain any points of $E[\boldsymbol{x}]$, since we chose a smooth connecting segment.

Equality of the functionals applies for the same reason as in the comparison of $\psi$ with $\phi$.

We will often switch between harmonic and curve coordinates, based on Lemmas 2.2 and 2.4. without further comment. We may also abandon the normalization conditions $\|\phi\|_{L^{2}}=1,\|\boldsymbol{\psi}\|_{L^{2}}=1$ and minimize the Rayleigh quotients $I[\boldsymbol{x}, \phi] /\|\phi\|_{L^{2}}^{2}, I[\boldsymbol{\psi}] /\|\boldsymbol{\psi}\|_{L^{2}}^{2}$ respectively. We may also abandon the length constraint and minimize (length $/ 2 \pi)^{2}$ times the Rayleigh quotient instead. 


\section{Existence AND PARTial Regularity For the RElaxed oval PROBlem}

We are now ready to prove

Theorem 3.1 (Existence and classification). The variational problem (2.6) has a minimum $\boldsymbol{\psi}$ with $I[\boldsymbol{\psi}] \leq 1$. The following alternative exists for the zero set $Z[\boldsymbol{\psi}]$ of such a minimizer:

Either (a) $Z[\boldsymbol{\psi}]=\emptyset$. In this case, the associated curve has everywhere defined curvature in the $L^{2}$ sense, i.e., $\boldsymbol{x} \in W^{2,2}$.

Or (b) $Z[\boldsymbol{\psi}]$ is a closed interval of length $<\pi$, possibly degenerating to a single point. In this case the weak loop condition is satisfied with equality, $\boldsymbol{x}$ contains a straight segment along $Z[\boldsymbol{\psi}]$ (omitted if $Z[\boldsymbol{\psi}]$ is a singleton), with $\phi \equiv 0$ on $Z[\boldsymbol{\psi}]$, and $\boldsymbol{x}$ has everywhere defined curvature in the $W_{l o c}^{2,2}\left(Z[\boldsymbol{\psi}]^{c}\right)$ sense.

Or (c1) $Z[\boldsymbol{\psi}]$ is a closed interval of length exactly $\pi$. In this case, the associated curve is a 'di-gon' (i.e., a line segment traversed once back and forth), with $\phi$ supported on one side of the di-gon.

Or $(\mathrm{c} 2) Z[\boldsymbol{\psi}]$ consists of two points with length exactly $\pi$ apart. In this case, the associated curve is the same 'di-gon', but $\phi$ is supported on both segments.

Definition 3.2. Minimizers in case (b) of the preceding theorem will be called D-shaped.

In the present section we will prove this theorem, and also show that minimizers must be convex planar curves. More specifically:

Theorem 3.3 (Planarity and convexity). Minimizers $\boldsymbol{\psi}$ of $(2.6)$ with $Z[\boldsymbol{\psi}]=\emptyset$ represent planar, strictly convex, real-analytic curves with strictly positive curvature.

$D$-shaped minimizers $\boldsymbol{\psi}$ (if any) represent planar convex curves, the portion over $Z[\boldsymbol{\psi}]^{c}$ of which is real-analytic and has strictly positive curvature.

However, it is worth announcing now the stronger result proved in Section 5 that case (b) of Theorem 3.1 does not occur:

Theorem 3.4 (Regularity). With the possible exception of the di-gon, minimizers for the variational problem (2.6) are smooth, and $Z[\psi]$ is empty.

The proof of this theorem will rely on the a-priori conclusions about hypothetical D-shaped minimizers that are proved in the present section.

Proof of Theorem [3.1. Assume $\left(\boldsymbol{\psi}_{n}\right)$ is a minimizing sequence, i.e., $I\left[\boldsymbol{\psi}_{n}\right] \rightarrow \inf I$, and hence bounded in $W^{1,2}$. We can extract a subsequence, again called $\left(\psi_{n}\right)$, that converges uniformly, and weakly in $W^{1,2}$, to a limit $\boldsymbol{\psi}_{*}$. We get $I\left[\boldsymbol{\psi}_{*}\right]=\int\left|\boldsymbol{\psi}_{*}^{\prime}\right|^{2} \leq$ $\lim \inf \int\left|\boldsymbol{\psi}_{n}^{\prime}\right|^{2}=\inf I$ as well as $\int\left|\boldsymbol{\psi}_{*}\right|^{2}=1$ routinely. We only need to show that $\boldsymbol{\psi}_{*}$ still satisfies the weak loop constraint. We find it convenient to tacitly adopt the practice of using the notation $\operatorname{sgn} \psi$ only in those cases where the possibility of $\boldsymbol{\psi}$ vanishing needs to be reckoned with, but to revert to $\boldsymbol{\psi} /|\boldsymbol{\psi}|$ in cases where this possibility has already been ruled out. We also abbreviate $Z\left[\boldsymbol{\psi}_{n}\right], Z\left[\boldsymbol{\psi}_{*}\right]$ as $Z_{n}, Z_{*}$ respectively.

Now clearly, on $Z_{*}^{c}$, it holds that $\operatorname{sgn} \boldsymbol{\psi}_{n} \rightarrow \boldsymbol{\psi}_{*} /\left|\boldsymbol{\psi}_{*}\right|$ pointwise, and trivially majorized. So we conclude

$$
\int_{Z_{*}^{c}} \operatorname{sgn} \boldsymbol{\psi}_{n} \rightarrow \int_{Z_{*}^{c}} \boldsymbol{\psi}_{*} /\left|\boldsymbol{\psi}_{*}\right| .
$$


On the other hand,

$$
\int_{Z_{*}^{c}} \operatorname{sgn} \boldsymbol{\psi}_{n}=\int_{Z_{*}^{c} \cap Z_{n}^{c}} \boldsymbol{\psi}_{n} /\left|\boldsymbol{\psi}_{n}\right|=\int_{Z_{n}^{c}} \boldsymbol{\psi}_{n} /\left|\boldsymbol{\psi}_{n}\right|-\int_{Z_{*} \backslash Z_{n}} \boldsymbol{\psi}_{n} /\left|\boldsymbol{\psi}_{n}\right|
$$

and therefore, using the fact that $\boldsymbol{\psi}_{n}$ satisfies the weak loop condition,

$$
\left|\int_{Z_{*}^{c}} \operatorname{sgn} \boldsymbol{\psi}_{n}\right| \leq\left|\int_{Z_{n}^{c}} \boldsymbol{\psi}_{n} /\right| \boldsymbol{\psi}_{n}||+\sigma\left(Z_{*} \backslash Z_{n}\right) \leq \sigma\left(Z_{n}\right)+\sigma\left(Z_{*} \backslash Z_{n}\right)=\sigma\left(Z_{*}\right) .
$$

By taking the limit on the left, using (3.1), we get $\left|\int_{Z_{*}^{c}} \boldsymbol{\psi}_{*} /\right| \boldsymbol{\psi}_{*}|| \leq \sigma\left(Z_{*}\right)$ as required.

The unit circle with constant eigenfunction, namely $\boldsymbol{\psi}(s)=(2 \pi)^{-1 / 2}\left[\begin{array}{l}\sin s \\ \cos s\end{array}\right]$ in $\mathbb{R}^{2}$ (or in $\mathbb{R}^{n}$, by imbedding), is an example with $I=1$, so clearly $\min I \leq 1$.

Having thus proved the existence of a minimizer, we now can get some limited regularity. Let $\boldsymbol{\psi}$ be a minimizer and $Z$ its zero set. Its complement $Z^{c}$, being open and nonempty, is either all of $S^{1}$ or is the union of finitely many or countably infinitely many intervals $J_{j}$ with respective lengths $\ell_{j}$.

With case (a) already being obvious from Lemma 2.4 let's look at the cases where $Z \neq \emptyset$. Restricting $\boldsymbol{\psi}$ to $J_{j}$, this interval contributes $R Q_{j}:=\int_{J_{j}}\left|\boldsymbol{\psi}^{\prime}\right|^{2} / \int_{J_{j}}|\boldsymbol{\psi}|^{2}$ to the Rayleigh quotient $R Q=\int_{0}^{2 \pi}\left|\psi^{\prime}\right|^{2} / \int_{0}^{2 \pi}|\boldsymbol{\psi}|^{2}$. As $R Q$ is a weighted average of the local Rayleigh quotients $R Q_{j}$, it could be lowered by changing $\boldsymbol{\psi}$ to $\mathbf{0}$ on $J_{j}$, if $R Q_{j}>R Q$. So for a minimizer $\boldsymbol{\psi}$, all local $R Q_{j}$ have to be equal, namely $=R Q[\boldsymbol{\psi}] \leq$ 1. But because of the Dirichlet BC's $\boldsymbol{\psi}=\mathbf{0}$ on $\partial I_{j}$, we know $R Q_{j} \geq\left(\pi / \ell_{j}\right)^{2}$, and so we conclude $\ell_{j} \geq \pi$. So we have either a single $J_{j}$ of length $\geq \pi$, or two $J_{j}$ 's of length exactly $\pi$. Now when $\sigma\left(J_{1}\right)=\sigma\left(J_{2}\right)=\pi$, then only $\kappa \equiv 0$ achieves a Rayleigh quotient 1 , which is an upper bound for the minimum. This leads to case (c2).

If $Z^{c}$ consists of a single interval $J_{1}$ of length $\pi$, the restriction $\left.\boldsymbol{x}\right|_{J_{1}}$ must still be a straight segment by the same reasoning, and the weak loop constraint forces $\left.\boldsymbol{x}\right|_{Z}$ to be a straight segment, too. So this is again the di-gon case in variant $(\mathrm{c} 1)$.

Now let $Z^{c}$ consist of a single interval of length $\left.\left.\in\right] \pi, 2 \pi\right]$, hence $Z$ is a closed interval of length $<\pi$, possibly degenerated to a point. Then $\boldsymbol{x}$ consists of a $W_{\text {loc }}^{2,2}$ curve segment parametrized over the closure of $Z^{c}$, with $\phi$ supported there, and another segment closing the curve, on which $\phi$ vanishes. If the weak loop constraint were satisfied with slack, we could shorten the total length by replacing the segment over $Z$ with a shorter straight segment, without changing either $I[\boldsymbol{x}, \phi]$ or $\|\phi\|_{L^{2}}$. Then dilating the curve $\boldsymbol{x}$ back to length $2 \pi$, and rescaling $\phi$ to unit $L^{2}$ norm, we would decrease $I[\boldsymbol{x}, \phi]$, contradicting the minimality of the original curve. So the weak loop constraint must have been satisfied with equality. By the strict triangle inequality, the segment over $Z$ must then be straight. This is case (b).

Introducing a Lagrange multiplier $\lambda$ for the normalization constraint $\int\left|\boldsymbol{\psi}^{\prime}\right|^{2}=1$ and a vector valued Lagrange multiplier $\mu$ for the loop constraint $\int \boldsymbol{\psi} /|\boldsymbol{\psi}|=\mathbf{0}$, we routinely get that a minimizer according to case (a) of Theorem 3.1 must satisfy the Euler Lagrange equation

$$
\boldsymbol{\psi}^{\prime \prime}+\lambda \boldsymbol{\psi}-(\mu \cdot \boldsymbol{\psi}) \boldsymbol{\psi} /|\boldsymbol{\psi}|^{3}+\mu /|\boldsymbol{\psi}|=0
$$

or, in other words,

$$
\boldsymbol{\psi}^{\prime \prime}+\lambda \boldsymbol{\psi}+\frac{1}{|\boldsymbol{\psi}|} \operatorname{pr}_{\boldsymbol{\psi} \perp} \mu=0
$$


where we have introduced the orthogonal projection of $\mu$ onto the orthocomplement of $\boldsymbol{\psi}$. A minimizer $\boldsymbol{\psi}_{*}$ according to case (b) in Theorem 3.1 would in particular minimize $I[\boldsymbol{\psi}]$ in the restricted class of those $\boldsymbol{\psi}$ for which $Z[\boldsymbol{\psi}]=Z\left[\boldsymbol{\psi}_{*}\right]=$ : $Z$ and $\int_{Z^{c}} \boldsymbol{\psi} /|\boldsymbol{\psi}|=\int_{Z^{c}} \boldsymbol{\psi}_{*} /\left|\boldsymbol{\psi}_{*}\right|$. So the same EL equations still hold in this case on the open interval $J:=Z^{c}$, together with the boundary conditions $\boldsymbol{\psi}=\mathbf{0}$ on $\partial J$. Stronger information about $\boldsymbol{\psi}$ near $\partial J$ will be obtained below, eventually ruling out case (b) altogether.

Testing the equation (3.2) with $\boldsymbol{\psi}$ shows that the Lagrange multiplier $\lambda$ for a minimizer $\boldsymbol{\psi}$ indeed coincides with the value $\lambda=\min I$.

As already observed in [3], equation (3.2) has a conserved quantity

$$
E=\frac{1}{2}\left|\boldsymbol{\psi}^{\prime}\right|^{2}+\frac{\lambda}{2}|\boldsymbol{\psi}|^{2}+\frac{\mu \cdot \boldsymbol{\psi}}{|\boldsymbol{\psi}|},
$$

and in higher dimensions than 2 , there are lower dimensional angular momenta arising from the rotation symmetry about the $\mu$ axis. The vector space spanned by $\boldsymbol{\psi}\left(s_{0}\right), \boldsymbol{\psi}^{\prime}\left(s_{0}\right)$ and $\mu$ is invariant, so solutions of the EL equations automatically remain in an at most 3 -dimensional subspace of $\mathbb{R}^{n}$; possible connecting straight segments in the case of D-shaped minimizers would not leave this space either. Therefore minimizers are curves in at most a 3-dimensional space, regardless of the dimension $n$ in which the Oval Problem was originally posed. It also follows from standard regularity results for ODEs that minimizers (or any extremals) are real-analytic curves on the complement of the zero set $Z[\boldsymbol{\psi}]$.

In the case of 2 dimensions, it is convenient to write the EL equations and the energy equation in polar coordinates, and to identify $\mathbb{R}^{2}$ with $\mathbb{C}$, where the real axis is chosen parallel to the vector $\mu$. It is therefore no loss of generality to choose $\mu \in$ $\mathbb{R}^{2} \cong \mathbb{C}$ to be a real nonnegative number $\mu$. Then, writing $\boldsymbol{\psi}(s)=R(s) \exp i \theta(s)$, the EL equations and energy become

$$
\begin{array}{ll}
R^{\prime \prime}+R\left(\lambda-\theta^{\prime 2}\right)=0 & \left(\text { note that } \theta^{\prime}=\kappa \text { and } R=\phi\right), \\
R \theta^{\prime \prime}+2 R^{\prime} \theta^{\prime}=\frac{\mu}{R} \sin \theta & \left(\text { can also be written as }\left(R^{2} \theta^{\prime}\right)^{\prime}=\mu \sin \theta\right), \\
E=\frac{1}{2}\left(R^{\prime 2}+R^{2} \theta^{\prime 2}+\lambda R^{2}\right)+\mu \cos \theta &
\end{array}
$$

The first equation returns the Schrödinger equation on the loop; the second equation describes the interaction between the Schrödinger eigenfunction and the curve that is necessary for an extremal.

The following simple geometric lemma will be useful in proving planarity of minimizers.

Lemma 3.5. Given a closed $C^{1}$ curve $\gamma: s \mapsto \boldsymbol{x}(s)$ in $\mathbb{R}^{n}$ (not necessarily injective), where $n \geq 3$, there exists a hyperplane $\Pi$ that is tangential to $\gamma$ at least twice, i.e., at points $\boldsymbol{x}\left(s_{1}\right), \boldsymbol{x}\left(s_{2}\right)$ with $s_{1} \neq s_{2}$.

Given a closed $C^{1}$ curve $\gamma: s \mapsto \boldsymbol{x}(s)$ in $\mathbb{R}^{2}$ (not necessarily injective), either there exists a line $\Pi$ that is tangential to $\gamma$ at least twice, or else the tangent angle $\theta$ is a strictly monotonic function of $s$ with $\theta(s+2 \pi)=\theta(s)+2 \pi$ or $\theta(s+2 \pi)=$ $\theta(s)-2 \pi$, with the sign depending on orientation.

While we doubt that this lemma would be new, we do not have a reference for it and provide a proof; actually we give two different proofs since both are interesting in their own right. Use of this lemma was inspired by Almut Burchard, and the proof by convexity uses her ideas. We only need the lemma for $n \leq 3$. 
Proof of Lemma 3.5 (by convexity). Let $K$ be the convex hull of $\gamma$. Every point in $K$ is a finite linear combination of curve points, and namely of at most $n+1$ points by Carathéodory's theorem. See for instance Ch. 17 of Rockafellar [5]. A consequence of Carathéodory's theorem is also that the convex hull of a compact set in $\mathbb{R}^{n}$ is compact; so $K$ is compact.

We first take care of the case $n \geq 3$. If the curve is not lying in a hyperplane already, $K$ is an $n$-dimensional convex body, whose boundary $\partial K$ therefore has Hausdorff dimension $n-1 \geq 2$. It cannot be filled by a $C^{1}$-curve, whose image has Hausdorff dimension 1. Therefore the boundary of $K$ contains a point $P$ that is not on the curve. Let the face $F$ be the intersection of $K$ with a supporting hyperplane $\Pi$ at $P$.

Now $P$ must be a convex combination of curve points $Q_{i}$. As $P \notin \gamma$, this cannot be the trivial convex combination, so $P$ cannot be an extreme point of $K$. Since $F$ is a face, the $Q_{i}$ (of which there are at least 2) must lie in $F$ as well (and $F$ has dimension at least 1 ). So we have found at least two curve points $Q_{i}$ lying in $F \subset \Pi$. The tangent vectors to $\gamma$ in the $Q_{i}$ lie in $\Pi$ because $\gamma$ lies on a single side of $\Pi$. This proves the $n \geq 3$ part of the lemma.

Now for the $n=2$ part, $K$ is a 2-dimensional convex compact set, therefore its boundary $\partial K$ is a simple closed Lipschitz curve. If $\partial K$ has a 1-dimensional face $F$, then $F$ is the convex hull of two distinct curve points $\boldsymbol{x}\left(s_{1}\right)$ and $\boldsymbol{x}\left(s_{2}\right)$, and the supporting line $\Pi$ through this face is a line of double tangency. As in the higher dimensional case, this happens in particular when $\partial K$ has a point that does not lie on $\gamma$.

In the other case, when there is no 1-dimensional face, $K$ is strictly convex, and every point of $\partial K \approx S^{1}$ is a curve point. If the continuous mapping $S^{1} \ni s \mapsto$ $\boldsymbol{x}(s) \in \partial K \subset \mathbb{R}^{2}$ fails to be injective, we again have a point of double tangency $\boldsymbol{x}\left(s_{1}\right)=\boldsymbol{x}\left(s_{2}\right)$ with $s_{1} \neq s_{2}$. However, if the mapping is injective, then it is a homeomorphism, and $\gamma$ is the boundary of the strictly convex set $K$. The claim about the monotonic dependence of $s$ on $\theta$ follows routinely from this.

Second Proof of Lemma 3.5 (via Borsuk-Ulam). Choose $\boldsymbol{n} \in S^{n-1}$ and maximize the continuous expression $\boldsymbol{x}(s) \cdot \boldsymbol{n}$ over $s \in S^{1}$ (compact). If $s_{0}$ is the location of a maximum, then the affine hyperplane $\Pi:=\boldsymbol{x}\left(s_{0}\right)+\{\boldsymbol{n}\}^{\perp}$ is tangential to $\gamma$ at $\boldsymbol{x}\left(s_{0}\right)$. Now assume the lemma is false. Then for each $\boldsymbol{n}$, the maximum of $\boldsymbol{x}(s) \cdot \boldsymbol{n}$ is taken on in a unique point. In other words, the function $f: S^{n-1} \rightarrow S^{1}, \boldsymbol{n} \mapsto$ $\arg \max (\boldsymbol{x}(s) \cdot \boldsymbol{n})$ is well defined. A routine argument implies that $f$ is continuous: Indeed, let $\boldsymbol{n}_{k} \rightarrow \boldsymbol{n}$ and consider the sequence $\left(f\left(\boldsymbol{n}_{k}\right)\right)$. If this sequence failed to converge to $f(\boldsymbol{n})$, we could extract a subsequence that stays bounded away from $f(\boldsymbol{n})$; but by compactness it would still have a further subsequence converging to some quantity $s_{*}$. Since $\boldsymbol{x}\left(f\left(\boldsymbol{n}_{k}\right)\right) \cdot \boldsymbol{n}_{k} \geq \boldsymbol{x}(s) \cdot \boldsymbol{n}_{k}$ for all $s$, we can pass to the limit and conclude $\boldsymbol{x}\left(s_{*}\right) \cdot \boldsymbol{n} \geq \boldsymbol{x}(s) \cdot \boldsymbol{n}$ for all $s \in S^{1}$. But this means $s_{*}$ is the (unique) $\arg \max \boldsymbol{x}(s) \cdot \boldsymbol{n}=f(\boldsymbol{n})$, which is a contradiction.

Now we focus on $n \geq 3$. By Borsuk-Ulam, a continuous function from $S^{n-1}$ to $\mathbb{R}^{n-1}$ must map some pair of antipodes into the same point. Using $n \geq 3$, we apply this to the function $f: S^{n-1} \rightarrow S^{1} \hookrightarrow \mathbb{R}^{n-1}$, obtaining a pair of antipodes $(\boldsymbol{n},-\boldsymbol{n})$ for which $f(\boldsymbol{n})=f(-\boldsymbol{n})=: s_{*}$. But this means $\max \boldsymbol{x}(s) \cdot \boldsymbol{n}=\min \boldsymbol{x}(s) \cdot \boldsymbol{n}=\boldsymbol{x}\left(s_{*}\right)$. $\boldsymbol{n}$, hence $\boldsymbol{x}$ lies entirely in the hyperplane $\Pi=\boldsymbol{x}\left(s_{*}\right)+\{\boldsymbol{n}\}^{\perp}$. This contradiction proves the lemma for $n \geq 3$. 
Now for $n=2$, we still have the continuous mapping $f: S^{1} \ni \boldsymbol{n} \mapsto \arg \max \boldsymbol{x}(s)$. $\boldsymbol{n} \in S^{1}$. We claim $f$ is injective. For if it were not, there would exist $s_{*}$ and two distinct vectors $\boldsymbol{n}_{1}, \boldsymbol{n}_{2}$ such that $\boldsymbol{x}(s) \cdot \boldsymbol{n}_{1} \leq \boldsymbol{x}\left(s_{*}\right) \cdot \boldsymbol{n}_{1}$ for all $s$, and likewise $\boldsymbol{x}(s) \cdot \boldsymbol{n}_{2} \leq \boldsymbol{x}\left(s_{*}\right) \cdot \boldsymbol{n}_{2}$. This would make $\boldsymbol{x}\left(s_{*}\right)$ a curve point in the vertex of a sector (smaller than a half plane) containing the entire curve. But this is impossible since $\boldsymbol{x}(\cdot)$ is $C^{1}$.

Now $f: S^{1} \rightarrow S^{1}$, being continuous and injective, is a homeomorphism, and $s$ is a monotonic function of the angle of $\boldsymbol{n}$, or equivalently, of the angle $\theta$ of the tangent vector, with $s(\theta+2 \pi)=s(\theta)+2 \pi$ or $s(\theta+2 \pi)=s(\theta)-2 \pi$ in the lift, depending on orientation.

Note that in the case of curves that are not imbedded but only immersed in $\mathbb{R}^{n}$ $(n \geq 3)$, the second proof guarantees the existence of 'two' tangency points $\boldsymbol{x}\left(s_{1}\right)$, $\boldsymbol{x}\left(s_{2}\right)$ with $s_{1} \neq s_{2}$, but does not rule out that this is a double point, $\boldsymbol{x}\left(s_{1}\right)=\boldsymbol{x}\left(s_{2}\right)$; the first proof asserts the slightly stronger statement $\boldsymbol{x}\left(s_{1}\right) \neq \boldsymbol{x}\left(s_{2}\right)$. The weaker version is the one we use. Both proofs construct a doubly tangent hyperplane $\Pi$ such that the entire curve lies on one side of $\Pi$. This latter property is not of essence for our purposes.

Proof of Theorem 3.3. We begin by showing planarity. For the case of a curve $\gamma$ with $Z[\boldsymbol{\psi}]=\emptyset$ (and without loss of generality in $\mathbb{R}^{3}$ ), we note first that $\gamma$ is realanalytic, as a solution to the EL equation. We use Lemma 3.5 directly to find a plane $\Pi$ tangential to $\gamma$ in two points $\boldsymbol{x}\left(s_{0}\right)$ and $\boldsymbol{x}\left(s_{1}\right)$. (We use only $s_{0} \neq s_{1}$, not necessarily $\boldsymbol{x}\left(s_{0}\right) \neq \boldsymbol{x}\left(s_{1}\right)$.) The points $s_{1}, s_{2}$ dissect $S^{1}$ into two open intervals $J_{1}, J_{2}$. We can now construct another curve $\tilde{\gamma}: s \mapsto \tilde{\boldsymbol{x}}(s)$ by letting $\tilde{\boldsymbol{x}}(s)=\boldsymbol{x}(s)$ for $s \in \bar{J}_{1}$ and $\tilde{\boldsymbol{x}}(s)=R_{\Pi} \boldsymbol{x}(s)$ for $s \in \bar{J}_{2}$, where $R_{\Pi}$ is the reflection in the plane $\Pi$. The new curve $\tilde{\gamma}$ is still admissible to (2.5); in particular it is still $C^{1}$ in $s_{1,2}$, even though its curvature may have jump discontinuities there. It will carry the same Schrödinger eigenfunction $\phi$. But since $I[\tilde{\boldsymbol{x}}, \phi]=I[\boldsymbol{x}, \phi], \gamma$ is still a minimizer and therefore $\boldsymbol{\psi}=\boldsymbol{x}^{\prime} \phi$ is a solution to the EL equations (3.2). By the unique continuation property for such solutions (or by analyticity in our case), $\gamma=\tilde{\gamma}$. In other words, $\left.\gamma\right|_{J_{2}} \subset \Pi$. The analogous argument can be made with the roles of $J_{1}$ and $J_{2}$ reversed; so $\gamma \subset \Pi$.

In the case of a D-shaped curve, we take one 'corner' point $\boldsymbol{x}\left(s_{2}\right)$ with $s_{2} \in Z[\boldsymbol{\psi}]$ and one regular point $\boldsymbol{x}\left(s_{1}\right)$ with $s \notin Z[\boldsymbol{\psi}]$. We define the plane $\Pi$ as passing through $\boldsymbol{x}\left(s_{1}\right)$ and $\boldsymbol{x}\left(s_{2}\right)$, and tangential to $\gamma$ at $s_{1}$. (In case $\boldsymbol{x}^{\prime}\left(s_{1}\right) \| \boldsymbol{x}\left(s_{2}\right)-\boldsymbol{x}\left(s_{1}\right)$, $\Pi$ is not unique, and any choice will serve the purpose.) We now define $\tilde{\gamma}$ as before. The plane $\Pi$ may intersect $\gamma, \tilde{\gamma}$ in other points, but this is of no concern. $\tilde{\gamma}$ is still admissible, and is still a minimizer. The unique continuation argument at $s_{1}$ guarantees that $\gamma$ coincides with $\tilde{\gamma}$ on the largest interval $J$ that contains $s_{1}$ and lies within $Z[\boldsymbol{\psi}]^{c}$. So that part of the curve is planar. But the remaining part is a straight segment connecting the endpoints of the curve segment $\boldsymbol{x}(\bar{J})$; so the entire curve is planar.

The same reflection argument now proves that a planar minimizer cannot have a double tangent, nor (in the case of a D-shaped minimizer) a tangent at a regular point $s_{1}$ that also passes through a point $\boldsymbol{x}\left(s_{2}\right)$ with $s_{2} \in Z[\boldsymbol{\psi}]$. According to the lemma, this implies for a regular minimizer (with $Z[\boldsymbol{\psi}]=\emptyset$ ) that $\theta$ is a strictly monotonic function of $s$. For a D-shaped minimizer, the first proof of Lemma 3.5 in the case $n=2$ still applies (as $C^{1}$ wasn't needed), giving that it is the boundary of 
a strictly convex set, and that the smooth part of the curve has the monotonicity property between $s$ and $\theta$.

Finally we want to argue the strengthened statement that the curvature is actually strictly positive (on the complement of $Z[\boldsymbol{\psi}]$ ). To this end, we use the EL equations in polar coordinates; see (3.4). We have seen that $s \mapsto \theta(s)$ is strictly monotonic; we can assume $\theta^{\prime}(s) \geq 0$ without loss of generality (else reflect the curve). We assume $\theta^{\prime}\left(s_{*}\right)=0$ for some $s_{*}$ and try to derive a contradiction.

This would make $s_{*}$ a minimum of $\theta^{\prime}$, and therefore $\theta^{\prime \prime}\left(s_{*}\right)=0$. From the second of the EL equations, $R \theta^{\prime \prime}+2 R^{\prime} \theta^{\prime}=\frac{\mu}{R} \sin \theta$, we infer $\mu \sin \theta\left(s_{*}\right)=0$. Now for the system of EL equations (3.4) with the initial conditions $\theta\left(s_{*}\right)=\theta_{0}$ (subject to $\left.\mu \sin \theta_{0}=0\right), \theta^{\prime}\left(s_{*}\right)=0, R\left(s_{*}\right)=R_{0} \neq 0, R^{\prime}\left(s_{*}\right)=R_{1}$, there exists one solution that can be calculated explicitly, namely $\theta(s) \equiv \theta_{0}$, and $R(s)$ solution to the constant coefficient problem $R^{\prime \prime}+\lambda R=0$ with the given initial conditions. By the uniqueness theorem for solutions to regular ODE initial value problems, this solution is the solution to the EL equation in question, i.e., $\theta$ is constant, contradicting the strict monotonicity.

This proves the theorem.

\section{Asymptotics for extremals near singularities}

This section, and the next, are devoted to the proof of Theorem 3.4

To avoid trivialities we note first that for a D-shaped minimizer, $\mu=\mathbf{0}$ is not possible. This is because (3.2) is trivial to solve for $\mu=\mathbf{0}$ : It gives $\boldsymbol{\psi}=\left[\begin{array}{l}a \cos (\omega s-\alpha) \\ b \cos (\omega s-\beta)\end{array}\right]$ with $\lambda=\omega^{2}$. Such a $\boldsymbol{\psi}$ can never vanish unless the two components are 'in phase'; but then the range of $\boldsymbol{\psi}$ would be 1-dimensional.

Our first step is to establish asymptotics near a singularity $(s=0$ with no loss of generality) for solutions to the EL equations.

Lemma 4.1. Suppose $R, \theta$ satisfy the EL equations (3.4) for $s \in] 0, \ell[$, with $R>0$ and $\theta^{\prime}>0$ there, and let $\mu \neq 0$. Suppose $R(s) \rightarrow 0$ and $\theta(s) \rightarrow \theta_{0}$ as $s \rightarrow 0$. Then the following conclusions hold:

(a) $\sin \theta_{0}=0$.

(b) $\lim _{s \rightarrow 0+} R^{\prime}(s)=:$ a exists and $a \geq 0$. Consequently $\lim _{s \rightarrow 0} \frac{R(s)}{s}=a$.

(c) $R(s) \theta^{\prime}(s) \rightarrow 0$ and $R^{2}(s) \theta^{\prime \prime}(s) \rightarrow 0$ as $s \rightarrow 0$.

Corollary 4.2. A D-shaped minimizer would have to be $C^{1}$.

Proof of the lemma. We use the assumption that $\lim _{s \rightarrow 0+} \theta(s)$ exists in the form that $\theta^{\prime}$ is integrable on $\left.] 0, \varepsilon\right]$.

From the energy estimate, it follows that $R^{\prime}$ is bounded, and therefore $R(s) \leq b s$ for some $b$. Also, $R^{2} \theta^{\prime}$ has a finite limit for $s \rightarrow 0+$, because it is an antiderivative, on $] 0, \ell[$, of the continuous function $\mu \sin \theta(s)$. If this limit were nonzero, we would conclude that $\theta^{\prime} \geq c / s^{2}$, which contradicts the fact that $\theta^{\prime}$ must be integrable.

Therefore we know $\lim _{s \rightarrow 0+} R^{2}(s) \theta^{\prime}(s)=0$. Again, as an antiderivative of $\mu \sin \theta(s)$, the function $R^{2} \theta^{\prime}$ is even $C^{1}$ on $[0, \ell[$, and this implies that the limit $\lim _{s \rightarrow 0+} R^{2}(s) \theta^{\prime}(s) / s$ exists and is finite. If this limit were nonzero, we would again get an estimate $\theta^{\prime} \geq c / s$, contradicting the integrability of $\theta^{\prime}$ near 0 . We have therefore proved $\lim _{s \rightarrow 0} \frac{R^{2}(s) \theta^{\prime}(s)}{s}=0$. 
Let us pretend to calculate this same limit in a different manner: the expression is of l'Hôpital type $0 / 0$, and $\lim _{s \rightarrow 0+} \frac{d\left(R^{2} \theta^{\prime}\right) / d s}{d s / d s}$ exists: it is $\lim _{s \rightarrow 0+}\left(R^{2} \theta^{\prime}\right)^{\prime}(s)=$ $\lim _{s \rightarrow 0+} \mu \sin \theta(s)=\mu \sin \theta_{0}$. Combining the two evaluations, we conclude $\sin \theta_{0}=$ 0 , proving part (a).

Next, $R \theta^{\prime}$ is bounded by the energy estimate and, with this, the Schrödinger equation $R^{\prime \prime}+R\left(\lambda-\theta^{\prime 2}\right)=0$ turns into an estimate $\left|R^{\prime \prime}\right| \leq b+c \theta^{\prime}$. Therefore $R^{\prime \prime}$ is integrable up to $s=0$, and we can extend $R^{\prime}$ continuously into 0 . We have thus proved part (b). Trivially $a \geq 0$. Below we will see that actually $a>0$.

Since we now know that $R^{\prime}(0)=a$ exists, the energy equation tells us that $R \theta^{\prime}$ has a limit as $s \rightarrow 0+$. If this limit were nonzero, we would again conclude $\theta^{\prime}(s)>c / s$, contradicting integrability. Now $R^{2} \theta^{\prime \prime}=\mu \sin \theta-2 R^{\prime} R \theta^{\prime}$ will go to 0 as $s \rightarrow 0$.

Proof of the corollary. Part (a) of the lemma implies the corollary. To see this, let us consider the EL equation (3.2) over the interval $Z[\boldsymbol{\psi}]^{c}$, which we assume to be ] $0, \ell\left[\right.$ with no loss of generality, and test it with $\left[\begin{array}{c}-\psi_{2} \\ \psi_{1}\end{array}\right]$. We obtain

$$
\left[\psi_{2}^{\prime} \psi_{1}-\psi_{1}^{\prime} \psi_{2}\right]_{0+}^{\ell-}+\int_{0}^{\ell} \mu \cdot\left[\begin{array}{c}
-\psi_{2} \\
\psi_{1}
\end{array}\right] /|\boldsymbol{\psi}| d s=0 .
$$

From the boundedness of $\boldsymbol{\psi}^{\prime}$ (energy theorem) and the vanishing of $\boldsymbol{\psi}$ on the boundary, we obtain that $\mu$ is orthogonal to $\int_{0}^{\ell}\left[\begin{array}{c}-\psi_{2} \\ \psi_{1}\end{array}\right] /|\boldsymbol{\psi}| d s$, hence parallel to $\int_{0}^{\ell} \boldsymbol{\psi} /|\boldsymbol{\psi}| d s=\boldsymbol{x}(\ell)-\boldsymbol{x}(0)$. On the other hand, in (3.4), $\theta$ was the angle between $\boldsymbol{\psi}$ (hence the curve tangent $\boldsymbol{x}^{\prime}$ ) and $\mu$ (recall $\boldsymbol{\mu} \neq \mathbf{0}$ ). So $\sin \theta_{0}=0$ means that the tangent vector $\boldsymbol{x}^{\prime}(s)$ becomes parallel to $\boldsymbol{x}(\ell)-\boldsymbol{x}(0)$ as $s \rightarrow 0$. This could be a $C^{1}$ curve, or a curve with a cusp (outward or inward pointing); but a convex curve cannot have cusps. So we always have $\boldsymbol{x}^{\prime}(0)$ in the opposite direction as $\boldsymbol{x}(\ell)-\boldsymbol{x}(0)$.

The cases $\theta_{0}=0$ and $\theta_{0}=\pi$ are equivalent under rotation of the curve by $\pi$, i.e., a shift of $\theta$ by $\pi$ (which could instead be absorbed in a sign change of $\mu$ ). However, if we have already chosen a preferred direction of $\mu$ (in the present hypothetical scenario), then the two cases are distinct:

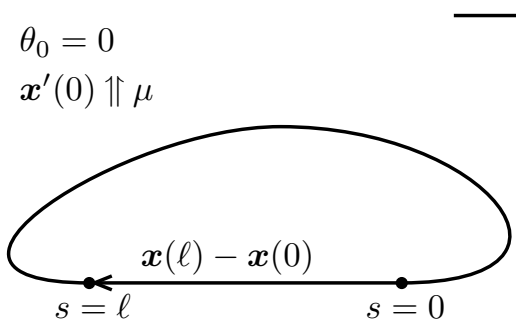

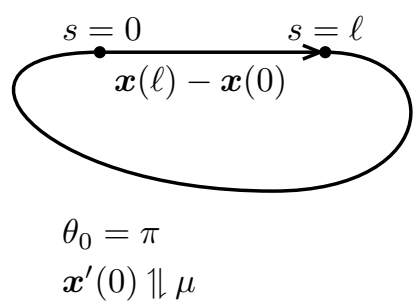

The key idea for finer asymptotics is that now the angular EL equation $R^{2} \theta^{\prime \prime}+$ $2 R R^{\prime} \theta^{\prime}=\mu \sin \theta$ has the same asymptotic behavior near $s=0, \theta=0$ as the Euler equation $a^{2} s^{2} \theta^{\prime \prime}+2 a^{2} s \theta^{\prime}=\mu \theta$, at least when $a \neq 0$. This similarity is brought out by a Sturm comparison argument, i.e., by integrating the derivative of a mixed Wronskian, made up of solutions of either equation. A variant of this same argument will show that indeed $a>0$. 
Lemma 4.3. Under the hypotheses of Lemma 4.1, and $\theta_{0}=0$, it follows that $a=R^{\prime}(0) \neq 0$.

Moreover, for $\mu>0$, i.e., the left case in (4.1), there exist constants $A>0$, $c>0$, such that the following finer asymptotics apply for $s \rightarrow 0+$ :

$$
\begin{aligned}
& \theta(s)=\frac{A}{c} s^{c}+O\left(s^{3 c}, s^{c+2}\right), \\
& \theta^{\prime}(s)=A s^{c-1}+O\left(s^{3 c-1}, s^{c+1}\right), \\
& R(s)=a s+\frac{a A^{2}}{2 c(2 c+1)} s^{2 c+1}-\frac{\lambda a}{6} s^{3}+o\left(s^{3}, s^{2 c+1}\right), \\
& R^{\prime}(s)=a+\frac{a A^{2}}{2 c} s^{2 c}-\frac{\lambda a}{2} s^{2}+o\left(s^{2}, s^{2 c}\right), \\
& R^{\prime \prime}(s)=a A^{2} s^{2 c-1}-\lambda a s+o\left(s, s^{2 c-1}\right) .
\end{aligned}
$$

The case $\mu<0$, i.e., the right case in (4.1), cannot occur.

Remark 4.4. For minimizers, it suffices to consider $c \leq \frac{1}{2}$ in the lemma. For otherwise, the curvature $\kappa=\theta^{\prime}$ would be square integrable, and the Rayleigh quotient for (2.5) could be improved by replacing $R \equiv \phi$ with $\max \{R, \varepsilon\}$, with the gain of order $\frac{\varepsilon}{a} \times a^{2}$ coming from $\int R^{\prime 2} d s$ and a smaller adverse term of order $o\left(\varepsilon^{2}\right)$ coming from $\int \kappa^{2} R^{2}$.

Proof of Lemma 4.3. We first assume $a>0, \theta_{0}=0, \mu>0$ and derive the claimed asymptotics. Thereafter, we will prove that $\mu<0$ leads to a contradiction. Finally we will lead $a=0$ to a contradiction.

We write the angular EL equation $\theta^{\prime \prime}+2 \frac{R^{\prime}}{R} \theta^{\prime}-\frac{\mu}{R^{2}} \sin \theta=0$ in the form

$$
s^{2} \theta^{\prime \prime}+2 s\left(1+T_{1}(s)\right) \theta^{\prime}-\left(\frac{\mu}{a^{2}}+T_{2}(s)\right) \theta=0,
$$

where

$T_{1}(s)=\frac{s R^{\prime}}{R}-1=o(1), \quad T_{2}(s)=\mu \frac{s^{2}}{R^{2}} \frac{\sin \theta}{\theta}-\frac{\mu}{a^{2}}=O\left(\theta^{2}\right)+O\left(\frac{s^{2}}{R^{2}}-\frac{1}{a^{2}}\right)=o(1)$.

For comparison, we consider the equation

$$
s^{2} u^{\prime \prime}+2 s u^{\prime}-\frac{\mu}{a^{2}} u=0
$$

with its solution $u(s)=s^{c}$, where $c=-\frac{1}{2}+\sqrt{\frac{1}{4}+\frac{\mu}{a^{2}}}$ is the positive root of the indicial equation $c(c-1)+2 c-\mu / a^{2}=0$. We integrate

$$
\left[s^{2}\left(\theta^{\prime} u-\theta u^{\prime}\right)\right]^{\prime}=\left(s^{2} \theta^{\prime \prime}+2 s \theta^{\prime}\right) u-\left(s^{2} u^{\prime \prime}+2 s u^{\prime}\right) \theta
$$

over $\left.\left[s_{1}, s_{2}\right] \subset\right] 0, \ell[$ and obtain

$$
\left[s^{2}\left(\theta^{\prime} u-\theta u^{\prime}\right)\right]_{s_{1}}^{s_{2}}=\int_{s_{1}}^{s_{2}}\left(T_{2} \theta-2 s T_{1} \theta^{\prime}\right) u d s=\int_{s_{1}}^{s_{2}} o(1)\left(|\theta u|+\left|s \theta^{\prime} u\right|\right) d s .
$$

We can let $s_{1} \rightarrow 0$ and obtain (using that $u, \theta, \theta^{\prime}>0$ )

$$
\left|s^{2}\left(\theta^{\prime} u-\theta u^{\prime}\right)\right| \leq \varepsilon \int_{0}^{s}\left(\theta+s \theta^{\prime}\right) u d s=\varepsilon s \theta u-\varepsilon \int_{0}^{s} s \theta u^{\prime} d s \leq \varepsilon s \theta u,
$$

where $\varepsilon$ can be made as small as we like, provided $s$ is chosen small. We will want $\varepsilon<c$. Dividing, we conclude

$$
\frac{u^{\prime}}{u}-\varepsilon \frac{1}{s} \leq \frac{\theta^{\prime}}{\theta} \leq \frac{u^{\prime}}{u}+\varepsilon \frac{1}{s}
$$


on some interval $] 0, \hat{s}]$. Integrating again over $\left.\left.\left[s_{1}, s_{2}\right] \subset\right] 0, \hat{s}\right]$, we get

$$
\frac{u\left(s_{2}\right)}{u\left(s_{1}\right)}\left(\frac{s_{2}}{s_{1}}\right)^{-\varepsilon} \leq \frac{\theta\left(s_{2}\right)}{\theta\left(s_{1}\right)} \leq \frac{u\left(s_{2}\right)}{u\left(s_{1}\right)}\left(\frac{s_{2}}{s_{1}}\right)^{\varepsilon} .
$$

Fixing $s_{2}$ and letting $s_{1}=: s$, this implies for $s<s_{2}$

$$
\frac{\theta(s)}{s^{c-\varepsilon}} \leq \frac{\theta\left(s_{2}\right)}{s_{2}^{c-\varepsilon}} \quad \text { and } \quad \frac{\theta(s)}{s^{c+\varepsilon}} \geq \frac{\theta\left(s_{2}\right)}{s_{2}^{c+\varepsilon}} .
$$

While this is not yet sufficient to establish the existence of $\lim \theta(s) / s^{c}$, we have at least shown that $\theta(s)=O\left(s^{c-\varepsilon}\right)$. Returning to the estimate for $\theta^{\prime} / \theta$, this implies also $\theta^{\prime}(s)=O\left(s^{c-1-\varepsilon}\right)$. This preliminary estimate will serve to improve the $o(1)$ terms in $R^{\prime}=a+o(1)$ and therefore in the Euler equation for $\theta$; redoing the Sturm comparison with the better estimate will then establish our desired estimate:

Namely the Schrödinger equation now tells us $\left|R^{\prime \prime}\right| \leq C s^{2 c-1-2 \varepsilon}$, hence $\mid R^{\prime}(s)-$ $R^{\prime}(0) \mid \leq C s^{2 c-2 \varepsilon}$ and $|R(s)-a s| \leq C s^{2 c-2 \varepsilon+1}$. So $\theta$ satisfies (4.2) with the improved estimates

$$
T_{1}(s)=O\left(s^{2 c-2 \varepsilon}\right), \quad T_{2}(s)=O\left(s^{2 c-2 \varepsilon}\right) .
$$

This improves our estimates (4.4), (4.5), (4.6) to

$$
\begin{gathered}
\left|s^{2}\left(\theta^{\prime} u-\theta u^{\prime}\right)\right| \leq C \int_{0}^{s}\left(\theta+s \theta^{\prime}\right) u s^{2 c-2 \varepsilon} d s \leq C s \theta u s^{2 c-2 \varepsilon}, \\
\frac{u^{\prime}}{u}-\frac{C}{s} s^{2 c-2 \varepsilon} \leq \frac{\theta^{\prime}}{\theta} \leq \frac{u^{\prime}}{u}+\frac{C}{s} s^{2 c-2 \varepsilon}, \\
\frac{u\left(s_{2}\right)}{u\left(s_{1}\right)}\left(1-C s_{2}^{2 c-2 \varepsilon}\right) \leq \frac{\theta\left(s_{2}\right)}{\theta\left(s_{1}\right)} \leq \frac{u\left(s_{2}\right)}{u\left(s_{1}\right)}\left(1+C s_{2}^{2 c-2 \varepsilon}\right) .
\end{gathered}
$$

The constant $C$ does not deteriorate as $s_{2} \rightarrow 0$. But for the moment we fix $s_{2}$ and conclude (with $s_{1}=: s$ ) that

$\limsup _{s \rightarrow 0} \frac{\theta(s)}{s^{c}} \leq \frac{\theta\left(s_{2}\right)}{s_{2}^{c}}\left(1-C s_{2}^{2 c-2 \varepsilon}\right)^{-1} \quad$ and $\quad \liminf _{s \rightarrow 0} \frac{\theta(s)}{s^{c}} \geq \frac{\theta\left(s_{2}\right)}{s_{2}^{c}}\left(1+C s_{2}^{2 c-2 \varepsilon}\right)^{-1}$.

Now we can let $s_{2} \rightarrow 0$ and find that $\lim _{s \rightarrow 0} \frac{\theta(s)}{s^{c}}$ exists. We will call this limit $A / c$, and it is positive because the lower bound for $\lim \inf \theta(s) / s^{c}$ was positive. Our estimate has also established that $\theta^{\prime} / \theta \sim c / s$. So we have found

$$
\theta(s) \sim \frac{A}{c} s^{c} \quad \text { and } \quad \theta^{\prime}(s) \sim A s^{c-1} .
$$

Inserting these in the Schrödinger equation already establishes the claimed asymptotics for $R^{\prime \prime}$, and by integration for $R^{\prime}, R$.

By feeding these asymptotics for $R$ back into the angular EL equation, we can get a quantitative error term for the asymptotics of $\theta, \theta^{\prime}$ :

$$
s^{2} \theta^{\prime \prime}+2 s \theta^{\prime}-\frac{\mu}{a^{2}} \theta=O\left(s^{2 c+1}, s^{3}\right) \theta^{\prime}+O\left(s^{2 c}, s^{2}\right) \theta=O\left(s^{3 c}, s^{2+c}\right) .
$$

Variation of constants quickly establishes $\theta(s)=\frac{A}{c} s^{c}+O\left(s^{3 c}, s^{c+2}\right)$ and $\theta^{\prime}(s)=$ $A s^{c-1}+O\left(s^{3 c-1}, s^{c+1}\right)$.

In the case $\mu<0$, the indicial equation has no positive roots. We compare with $u=s^{c}$ where either $c=-\frac{1}{2}+\sqrt{\frac{1}{4}+\frac{\mu}{a^{2}}} \in\left[-\frac{1}{2}, 0\left[\right.\right.$ (for $\frac{\mu}{a^{2}} \geq-\frac{1}{4}$ ), or $c=-\frac{1}{2}$ (for 
$\left.\mu<-\frac{1}{4}\right)$. Then instead of (4.4), we obtain

$$
\begin{aligned}
{\left[s^{2}\left(\theta^{\prime} u-\theta u^{\prime}\right)\right]_{s_{1}}^{s_{2}} } & \left.=\int_{s_{1}}^{s_{2}}\left(\left(\min \left\{0, \frac{\mu}{a^{2}}-\frac{1}{4}\right\}+T_{2}\right) \theta-2 s T_{1} \theta^{\prime}\right) u\right) d s \\
& \leq \int_{s_{1}}^{s_{2}} o(1)\left(|\theta u|+\left|s \theta^{\prime} u\right|\right) d s,
\end{aligned}
$$

and from this the one-sided estimate

$$
s^{2} \theta^{\prime} u-s^{2} \theta u^{\prime} \leq \varepsilon s \theta u .
$$

This implies $\frac{\theta^{\prime}}{\theta} \leq \frac{u^{\prime}}{u}+\frac{\varepsilon}{s}=\frac{c+\varepsilon}{s}<0$, contradicting the hypothesis $\theta^{\prime}>0$.

Finally, we consider the case $a=0$. Letting $s \rightarrow 0$ in the energy theorem, we infer that $E=\mu$, and we write the energy theorem as

$$
\mu(1-\cos \theta)=\frac{1}{2}\left(R^{\prime 2}+R^{2} \theta^{\prime 2}+\lambda R^{2}\right) .
$$

The case $\mu<0$ immediately forces $R \equiv 0, \cos \theta \equiv 1$, since the two sides have opposite signs. In the case $\mu>0$ and $a=0$, we can proceed similarly as we did for $a>0$, but with an iterative improvement of the estimate that eventually implies $R(s) \equiv 0$ on some interval $\left[0, s_{*}\right]$, a contradiction. We assume $\mu=1$, which is no loss of generality, because we can make $R / \sqrt{\mu}$ into our new function $R$. To begin with, $E=\mu=1$ implies $R^{\prime 2} \leq 2(1-\cos \theta)=4 \sin ^{2}(\theta / 2)$, hence $\left|R^{\prime}\right| \leq \theta$.

Let us choose $a_{0}:=\frac{1}{2}$ and $s_{*}$ so that $\theta\left(s_{*}\right) \leq \frac{1}{4}$ and also $\left|R^{\prime}\right| \leq \frac{1}{2} a_{0}$ on $\left[0, s_{*}\right]$. The latter is possible since $R^{\prime} \rightarrow 0$ as $s \rightarrow 0$. We want to prove inductively $R \leq \frac{1}{2} a_{n} s$ on $\left[0, s_{*}\right]$ for a sequence $a_{n} \rightarrow 0$. The start of the induction for $n=0$ follows trivially from integrating $\left|R^{\prime}\right| \leq \frac{1}{2} a_{0}$.

Towards an induction step, we use the Sturm comparison argument for a onesided estimate on $\theta$. Given $a_{n} \leq \frac{1}{2}$, we let $c_{n}$ be the positive solution to $c(c-1) a_{n}^{2}+$ $c a_{n}=\mu=1$, namely

$$
c_{n}=\frac{-a_{n}+a_{n}^{2}+\sqrt{\left(a_{n}-a_{n}^{2}\right)^{2}+4 a_{n}^{2}}}{2 a_{n}^{2}},
$$

and $u_{n}:=s^{c_{n}}$. Note that $a_{n} \leq \frac{1}{2}$ implies $c_{n}>1$. Then (dropping the subscript $n$ for a moment)

$$
\begin{aligned}
{\left[R^{2}\left(\theta^{\prime} u-\theta u^{\prime}\right)\right]^{\prime} } & =\left(R^{2} \theta^{\prime \prime}+2 R R^{\prime} \theta^{\prime}\right) u-\left(R^{2} u^{\prime \prime}+2 R R^{\prime} u^{\prime}\right) \theta \\
& =\sin \theta u-\left(\frac{R^{2} c(c-1)}{s^{2}}+\frac{2 R R^{\prime} c}{s}\right) u \theta .
\end{aligned}
$$

Using $|R| \leq \frac{1}{2} a_{n} s$ and $\left|R^{\prime}\right| \leq \frac{1}{2} a_{0} s=\frac{1}{4} s$, the final parenthesis is $\leq \frac{1}{4}$, so the right hand side is $\geq\left(\frac{2}{\pi}-\frac{1}{4}\right) \theta u>0$. So we have $\left[R^{2}\left(\theta^{\prime} u-\theta u^{\prime}\right)\right]^{\prime} \geq 0$ on $\left[0, s_{*}\right]$. Integrating from 0 to $s$, we have $\theta^{\prime} u-\theta u^{\prime} \geq 0$, hence $\theta^{\prime} / \theta \geq c / s$ on $\left.] 0, s_{*}\right]$. Integrating again from $s$ to $s_{*}$, we have $\theta(s) \leq \theta\left(s_{*}\right)\left(\frac{s}{s_{*}}\right)^{c}$. With the inequality $\left|R^{\prime}\right| \leq \theta$ from the energy estimate, we infer

$$
\left|R^{\prime}\right| \leq \theta\left(s_{*}\right)\left(\frac{s}{s_{*}}\right)^{c} \quad \text { hence } \quad|R| \leq \frac{1}{c+1} \theta\left(s_{*}\right)\left(\frac{s}{s_{*}}\right)^{c+1} s_{*} \leq \frac{1}{c} \theta\left(s_{*}\right) s \leq \frac{1}{2} \frac{1}{2 c} s .
$$

Therefore, assuming $R(s) \leq \frac{1}{2} a_{n} s$ with $a_{n} \leq \frac{1}{2}$, and $\left|R^{\prime}(s)\right| \leq \frac{1}{2} a_{0}=\frac{1}{4}$, we have concluded $R(s) \leq \frac{1}{2} a_{n+1} s$, with

$$
a_{n+1}=1 /\left(2 c_{n}\right)=\frac{a_{n}^{2}}{a_{n}^{2}-a_{n}+\sqrt{4 a_{n}^{2}+\left(a_{n}-a_{n}^{2}\right)^{2}}} \leq \frac{a_{n}^{2}}{a_{n}^{2}-a_{n}+2 a_{n}}=\frac{a_{n}}{a_{n}+1} .
$$


The positive sequence $\left(a_{n}\right)$ is therefore decreasing and has a limit, which has to satisfy $0 \leq a \leq \frac{a}{a+1}$, hence $a=0$. Thus $R \equiv 0$ on $\left[0, s_{*}\right]$; this contradiction rules out $a=0$.

\section{NoneXistence OF D-Shaped Minimizers}

We are now ready to complete the proof of Theorem 3.4 .

Proof of Theorem 3.4. Assume we have an extremal $\gamma$ consisting of a strictly convex curve $(x(s), y(s))$ for $s \in[0, \ell]$, with $(x(0), y(0))=(0,0),(x(\ell), y(\ell))=(\ell-2 \pi, 0)$ and $\left(x^{\prime}(0), y^{\prime}(0)\right)=(1,0)=\left(x^{\prime}(\ell), y^{\prime}(\ell)\right)$, and of the straight segment $[-(2 \pi-\ell), 0]$ on the $x$-axis. This is understood to include the case $\ell=2 \pi$. According to the asymptotics obtained, we may assume that we have $R(s) \sim a s$ for $s \rightarrow 0+$, with $a>0$, and all the finer results from Lemma 4.3. As mentioned before, we may and will also assume $c \leq \frac{1}{2}$, which means that the curvature $\kappa(s) \rightarrow \infty$ in a nonsquare-integrable manner as $s \rightarrow 0+$. This simplifies the mixed-power error terms in Lemma 4.3.

We show that such an extremal cannot be a minimizer by giving an explicit variation that lowers the eigenvalue. The variation we give is a strong variation, in the sense that, while the change in $\theta$ is small, the change in $\theta^{\prime}$ is not. This type of variation does not enter in the derivation of the EL equation and provides therefore new information.

Basically, we connect $\boldsymbol{x}(0)$ and $\boldsymbol{x}(\sigma)$, for small $\sigma$ 's, with a comparison curve that preserves the $C^{1}$ regularity, but whose curvature stays bounded; and we keep the eigenfunction $R$ constant on this segment. The analogous change is made on the interval $s \in\left[-\sigma^{\prime}+\ell, \ell\right]$, where we insist that $R(\sigma)=R\left(-\sigma^{\prime}-\ell\right)=: R_{0}$.

Notice that we have the same $a$ at both sides of the segment, since $a$ is determined by the energy theorem $E=\frac{1}{2} a^{2}+\mu$. In view of the local asymptotics $R \sim a s$, this ensures that $\sigma^{\prime} / \sigma \rightarrow 1$ as $\sigma \rightarrow 0$.

The mentioned change in the curve $\boldsymbol{x}(\cdot)$ may have a slight effect on the length, which we correct by scaling.

So we strive to connect the points $(0,0)$ (with horizontal tangent) to the point $B=\left(x_{0}, y_{0}\right)$ with slope $\left.\frac{d y}{d x}\right|_{B}=m$. We do this by means of a cubic spline Spl given as $y=k_{2} x^{2}+k_{3} x^{3}$ with $k_{2}=\left(3 y_{0}-m x_{0}\right) / x_{0}^{2}, k_{3}=\left(m x_{0}-2 y_{0}\right) / x_{0}^{3}$.

Specifically in our case,

$$
\left[\begin{array}{l}
x_{0} \\
y_{0}
\end{array}\right]=\gamma(\sigma)=\left[\begin{array}{l}
\int_{0}^{\sigma} \cos \theta(s) d s \\
\int_{0}^{\sigma} \sin \theta(s) d s
\end{array}\right]=\left[\begin{array}{l}
\sigma-\frac{A^{2}}{2 c^{2}(2 c+1)} \sigma^{2 c+1}+O\left(\sigma^{4 c+1}\right) \\
\frac{A}{c(c+1)} \sigma^{c+1}+O\left(\sigma^{3 c+1}\right)
\end{array}\right],
$$

and

$$
m=\arctan \theta(\sigma)=\frac{A}{c} \sigma^{c}+O\left(\sigma^{3 c}\right),
$$

and therefore $k_{2}=\frac{2-c}{c(c+1)} A \sigma^{c-1}+O\left(\sigma^{3 c-1}\right)$ and $k_{3}=\frac{c-1}{c(c+1)} A \sigma^{c-2}+O\left(\sigma^{3 c-2}\right)$. The length of this spline is $\int_{0}^{x_{0}} \sqrt{1+y^{\prime 2}} d x=\left(1+O\left(\sigma^{2 c}\right)\right)\left(\sigma-O\left(\sigma^{2 c+1}\right)\right)$, i.e., it differs from the length $\sigma$ of the original curve piece by at most $O\left(\sigma^{2 c+1}\right)$.

The curvature $\tilde{\kappa}$ of the spline is

$$
\begin{aligned}
\tilde{\kappa} & =\frac{y^{\prime \prime}}{\left(1+y^{\prime 2}\right)^{3 / 2}}=\frac{2 k_{2}+6 k_{3} x}{\left(1+\left(2 k_{2} x+3 k_{3} x^{2}\right)^{2}\right)^{3 / 2}} \\
& =\left(2 k_{2}+6 k_{3} s\right)\left(1+O\left(\sigma^{2 c}\right)\right)=O\left(\sigma^{c-1}\right) .
\end{aligned}
$$


Therefore $\int_{\mathrm{Spl}} \tilde{\kappa}(s)^{2} d s R(\sigma)^{2}=O\left(\sigma^{2 c-1}\right) \sigma^{2}=O\left(\sigma^{2 c+1}\right)$.

The new curve $\tilde{\gamma}$ consists of the cubic spline Spl just constructed, the old curve segment $\left.\gamma\right|_{\left[\sigma, \ell-\sigma^{\prime}\right]}$, an analogous cubic spline $\operatorname{Spl}^{\prime}$ connecting $\left(x\left(\ell-\sigma^{\prime}\right), y\left(\ell-\sigma^{\prime}\right)\right)$ to $(x(\ell), y(\ell))=(\ell-2 \pi, 0)$, and the straight segment $[\ell-2 \pi, 0]$ on the $x$-axis. We consider a new function $\tilde{R}$ on $\tilde{\gamma}$ that coincides with $R$ on $\left[\sigma, \ell-\sigma^{\prime}\right]$ and is constant $R_{0}=R(\sigma)=R\left(\ell-\sigma^{\prime}\right)$ otherwise. Then

$$
\begin{aligned}
\int_{\tilde{\gamma}}\left(\tilde{R}^{2} \tilde{\kappa}^{2}+\tilde{R}^{\prime 2}\right) d s-\int_{\gamma}\left(R^{2} \kappa^{2}+R^{\prime 2}\right) d s \\
\leq\left(\int_{\mathrm{Spl}}+\int_{\mathrm{Spl}^{\prime}}\right) \tilde{R}^{2} \tilde{\kappa}^{2} d s-\left(\int_{0}^{\sigma}+\int_{\ell-\sigma^{\prime}}^{\ell}\right) R^{\prime 2} d s \\
\leq O\left(\sigma^{2 c+1}\right)-a^{2}\left(\sigma+\sigma^{\prime}\right)=-2 a^{2} \sigma+O\left(\sigma^{2 c+1}\right) .
\end{aligned}
$$

Likewise

$$
\begin{aligned}
\int_{\tilde{\gamma}} \tilde{R}^{2} d s & -\int_{\gamma} R^{2} d s \geq(2 \pi-\ell) R_{0}^{2}+R_{0}^{2}\left(\int_{\mathrm{Spl}}+\int_{\mathrm{Spl}^{\prime}}\right) d s-\left(\int_{0}^{\sigma}+\int_{\ell-\sigma^{\prime}}^{\ell}\right) R^{2} d s \\
\geq & (2 \pi-\ell) a^{2} \sigma^{2}+a^{2} \sigma^{2}\left(\sigma+\sigma^{\prime}+O\left(\sigma^{1+2 c}\right)\right)-\frac{a^{2}}{3} \sigma^{3}-\frac{a^{2}}{3} \sigma^{\prime 3} \\
\geq & (2 \pi-\ell) a^{2} \sigma^{2}+\frac{4 a^{2}}{3} \sigma^{3}-O\left(\sigma^{3+2 c}\right) \geq 0 .
\end{aligned}
$$

Therefore

$$
R Q[\tilde{\gamma}] \leq R Q[\gamma]-\frac{2 a^{2} \sigma}{\int_{\gamma} R^{2} d s}+O\left(\sigma^{2 c+1}\right) \leq \lambda-b \sigma
$$

for some $b>0$. Rescaling $\tilde{\gamma}$ to original length introduces a factor $(L[\tilde{\gamma}] / 2 \pi)^{2} \leq$ $1+O\left(\sigma^{2 c+1}\right)$, which still leaves us with a competitor whose Rayleigh quotient is below $\lambda$.

This proves that a D-shaped extremal cannot be minimal.

\section{REFERENCES}

[1] Rafael D. Benguria and Michael Loss, Connection between the Lieb-Thirring conjecture for Schrödinger operators and an isoperimetric problem for ovals on the plane, Partial differential equations and inverse problems, Contemp. Math., vol. 362, Amer. Math. Soc., Providence, RI, 2004, pp. 53-61, DOI 10.1090/conm/362/06604. MR2091490 (2005f:81057)

[2] Jacob Bernstein and Christine Breiner, A variational characterization of the catenoid, Calc. Var. Partial Differential Equations 49 (2014), no. 1-2, 215-232, DOI 10.1007/s00526-012-0579z. MR 3148113

[3] Almut Burchard and Lawrence E. Thomas, On an isoperimetric inequality for a Schrödinger operator depending on the curvature of a loop, J. Geom. Anal. 15 (2005), no. 4, 543-563, DOI 10.1007/BF02922244. MR2203162 (2007c:58019)

[4] Helmut Linde, A lower bound for the ground state energy of a Schrödinger operator on a loop, Proc. Amer. Math. Soc. 134 (2006), no. 12, 3629-3635 (electronic), DOI 10.1090/S0002-993906-08483-8. MR2240676 (2007m:81082)

[5] R. Tyrrell Rockafellar, Convex analysis, Princeton Mathematical Series, No. 28, Princeton University Press, Princeton, N.J., 1970. MR0274683 (43 \#445)

Department of Mathematics, University of Tennessee, Knoxville, Tennessee 37996

E-mail address: denzler@math.utk.edu 